\title{
Traffic congestion mitigation: combining engineering and economic perspectives
}

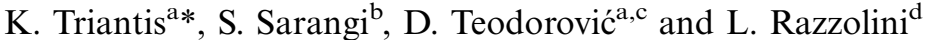 \\ ${ }^{a}$ Department of Industrial and Systems Engineering, Virginia Polytechnic Institute and State \\ University, 7054 Haycock Road, Falls Church, VA 22043, USA; ${ }^{b}$ Department of Economics, \\ Louisiana State University, Baton Rouge, LA 70803, USA; ${ }^{c}$ Faculty of Transport and Traffic \\ Engineering, University of Belgrade, Vojvode Stepe 305, 11000 Belgrade, Serbia; ${ }^{d}$ Department of \\ Economics, Virginia Commonwealth University, Richmond, VA 23284, USA
}

(Received 6 September 2010; accepted 16 April 2011)

\begin{abstract}
This paper introduces and provides a context for a special issue consisting of five selected papers that examine traffic congestion mitigation, with a focus on combining engineering and economic perspectives. Each paper provides novel insights of their own. The papers cover the modeling of parking behavior using possibility theory as well as the evaluation of a novel concept called Highway Space Inventory Control, where drivers must book in advance for their highway usage. A further paper evaluates the implications of new types of traffic pricing schemes and the challenges they face. Another paper tackles exactly this problem by evaluating different measures using data from the Netherlands. A fourth paper examines the implications of a disaster (bridge collapse) on traffic and assigns an economic value to such an outcome. The final paper is a case study that shows that price-based mechanisms may not be the best way to curb congestion.
\end{abstract}

Keywords: traffic congestion; mitigation; engineering; economics

\section{Introduction and context}

Congestion and queuing are now parts of our everyday life with examples aplenty. Pedestrians usually wait before crossing the street until traffic has stopped from both directions. Big airports are often busy and aircraft are put into a holding pattern with other aircraft waiting to land. Queuing time for security passenger search has become very high at many airports. Vessel bunching causes significant port congestion. Trucks wait in line at ports to pick-up a loaded container or to dropoff an empty container. When entering a tall building we press a button and we wait for an elevator. Our waiting time depends on the number of other requests for the elevator, as well as on the number of elevators. Every day thousands of vehicles are delayed waiting for a traffic signal. Callers call specific telephone numbers expecting that there will be an operator to answer their call. In many cases they have to wait a while before they can talk with an operator. The consequences of congestion are delay, compromised levels of service, and/or discomfort.

Why do we wait? The answer is simple: Whenever demand for a resource exceeds capacity this manifests itself as a queue. This can occur in two different ways. First, if

*Corresponding author. Email: triantis@vt.edu 
supply is woefully short then demand will always exceed supply, leading to permanent queues. Second, queuing may be a permanent feature but may arise periodically. A lot of customers (pedestrians, drivers, cars, aircraft, vessels, etc.) request various services on a daily basis (crossing the street, passing through the intersection, landing on runway, unloading at the dock, etc.). Service demand is characterized by the customer arrival rate. Arrival rates vary significantly depending on time of day, day in a week, or month in a year. The service rate indicates the number of customers who could be served in a time unit. Obviously, there are fluctuations in arrival rates and service times in many queuing systems. These fluctuations create queues and decrease the level of service offered to clients.

One of the most significant day-to-day queues manifest themselves on highways. An increasing number of US highways and roads experience very significant traffic congestion problems. On the other hand, physical and safety conditions on most Interstate highways have been improving. Based on congestion trends for 439 selected areas from 1982 to 2007, the Texas Transportation Institute (TTI) reports that the costs of traffic congestion for Americans is $\$ 87.2$ billion (in constant 2007 dollars) in wasted time and fuel annually (Schrank and Lomax 2009). Many urban areas in the world including but not limited to London, Paris, Stockholm, Tokyo, and Beijing have overwhelming traffic congestion that causes significant economic losses. Recently, innovative solutions such as congestion pricing have been implemented in some areas to fight congestion. Nevertheless, the evaluation of the impacts of such new interventions needs to be done.

The solution to the waiting problem can be achieved in two ways - either through the supply side or the demand side. In order to achieve low growth in traffic congestion in cities, the rate of increase of supply should match the rate of increase in demand. However, expanding traffic network capacities by building more roads and other infrastructure is extremely costly as well as environmentally damaging. Furthermore, and as explained in Section 3 of this introduction, simply adding capacity leads to opposite effects than those originally anticipated. Hence we have frequently relied on the second method, that is the demand side of the problem, by making people pay more for the scarce resource. Relying on the idea that time is money, longer waiting times have been used as the price of the scarce resource. An alternative idea that is increasingly gaining currency is to utilize the existing supply more efficiently by incentivizing users. More efficient usage of the existing supply, for example by spreading the peak demand onto off-peak hours, is vital in order to sustain the growing travel demand.

Travel Demand Management (TDM) or incentive-based techniques involving various strategies that increase travel choices to consumers have been proposed by researchers, planners, and transportation professionals. TDM or the incentive-based approach helps create a well-balanced, less automobile-dependent transportation system (VTPI 2011). TDM strategies include alternative mode encouragement strategies like 'Park-and-ride facilities'; 'High Occupancy Vehicle (HOV) facilities'; 'Transit service improvements and Transit Payment innovations'; 'Ridesharing programs'; 'Bicycle facility and Pedestrian facility improvements'; 'Telecommuting'; 'Alternative work hours'; driving disincentives like 'Congestion Pricing,' 'Increased fuel tax/mile fee,' 'Vehicle restrictions and Traffic calming'; parking programs such as 
'Increased and marginal parking price,' 'Preferential parking to rideshare vehicles,' 'Parking enforcement'; and others.

The traffic demand management or incentive-based approaches that have been mentioned thus far have relied on combinations of engineering approaches that have been used to formulate and evaluate the proposed strategies. It is the contention of this special issue that these methodological approaches require that behavioral and social considerations should be incorporated so as to formulate robust policies for traffic congestion mitigation.

A fundamental hypothesis is that the level of traffic congestion depends on the individual social interactions of drivers/co-drivers/passengers, as well as on the policy choices made by the traffic authorities. When dealing with traffic congestion, engineering, economic, and social issues need to be considered simultaneously, in conjunction with the assumption that the individual agents (drivers, passengers, transit operators, and traffic authorities) have the autonomy to make decisions, communicate, and interact with one another. Relatively minor changes in individual behavior can cause significant changes in both the traffic and the social networks affecting both route choices and social interaction. In other words, complex collective behavior can emerge from simple actions of individual agents. Traffic congestion can be viewed as an emergent phenomenon that is often difficult to predict and that is sometimes even counterintuitive.

One can analyze traffic congestion using integrated analytical approaches that allow for different views (transportation agencies, consumer, and society). A key short-term objective of these approaches is to find an optimal mix of dynamic demand-based management strategies that can take advantage of the newly available intelligent transportation technologies. The notion of 'optimal' involves the use of strategies that spread traffic congestion both in space and time, so that the social cost of congestion is minimized. In addition, to study the long-term social costs of congestion one can propose to use dynamic modeling that goes beyond pricing of roads and compares, instead, driving to the use of alternative transportation modes.

In the remaining sections of this introduction, we briefly summarize engineering approaches that also rely on economic principles and on economic approaches that require engineering fundamentals. We then conclude with an overview of the five papers that are part of this special issue.

\section{Engineering research with linkages to economics}

It is very difficult to contemplate engineering approaches that are designed to mitigate traffic congestion that do not consider economic fundamentals. This in part is due to the linkage between the proposed engineering solutions and their impact on the associated costs caused by traffic congestion. First, there are time costs such as increased average travel time and unexpected delays. Second, one can identify physical costs such as fuel costs and depreciation of vehicles. Third, there are environmental costs associated with noise and air pollution. Last but not least, one of the unintended consequences of traffic congestion is that it is very detrimental to community life. As Putnam (2000) has expressed it, in round numbers, the evidence suggests that each additional 10 minutes in daily commuting time cuts involvement in community affairs by $10 \%$. 
Furthermore, one needs to consider the impact of the work of many economists that have studied the effects of road pricing. Road user pricing as a means of mitigating congestion dates back to the seminal paper by Dupuit (1844). Thereafter, in the early 1920s, economists Pigou (1920) and Knight (1924) continued to enhance the theory of congestion pricing. In the 1960s, a resurrection of research interest in congestion pricing appeared thanks to the initiative of Nobel Economist laureate William Vickrey (1969). It is known, for example, that drivers and passengers perceive only their own travel time costs. They do not take into account the additional delay their trips impose on other passengers. Noticing these facts, Vickrey claimed that charges should reflect to the extent that this is possible, the marginal social cost of each trip in terms of the impact on others. In other words, it seems very logical that users should pay a price equivalent to the delay cost they impose on others. In this way, travel patterns probably could be changed. A probable consequence could be the efficiency improvement of transportation capacity utilization, and the probable reduction of future transportation capacity requirements.

Simultaneously, publication in the UK of the Smeed Report (1964) also boosted the resurgence in congestion pricing research. The foreword states that 'charges would be in the nature of prices for using the roads, the prices varying from one place and time to another according to the costs, notably the congestion costs, involved in driving in a particular area at a particular time.' It also indicates that 'when a pricing system is used on the roads, a useful general rule upon which to base prices is that the road user should pay a sum equal to the costs he imposes upon others' (Smeed Report 1964, p. 2).

Owing to the rapidly increased traffic congestion resulting from the growth of economic activities and declining revenue generated from motor fuel taxes related to travel, application of congestion pricing accelerated in the 1980s and 1990s. Many schemes were attempted during this time. Although some failed to be implemented after deliberate planning, a few schemes have been successfully implemented up to now, for example the practices of congestion pricing found in London (Transport for London 2008) and Stockholm (Transportation Research Part A 2009) among others.

Additionally, many researchers in engineering have been experimenting with various approaches that rely on Intelligent Transportation Systems (ITS). For example, the advancement of Electronic Payment Systems (EPS) has made it possible for planners and researchers to conceive of dynamic TDM strategies. Another example is the paper by Ottomanelli et al. in this special issue where the authors provide an example of how an engineering approach grounded in possibility theory can offer an evaluation of parking users' behavior. Another novel demand management idea that is being explored by Edara $e t$ al. as part of this special issue is the notion of Highway Space Inventory Control. Under this strategy drivers are required to reserve highway space in advance, just like aircraft arriving at and departing from airports, and the price of highway space will vary with the date and time of purchase. Those without reservations will not have access to the road. Similar notions have been explored by Teodorović and Lučić (2005) and Zhao et al. (2010) who investigate the notions of garage reservation and downtown space reservation systems, respectively. The implementation of these notions requires that the necessary technology is available and that the notion of social welfare, a core economic issue, 
is fully explored. This means that the social and economic impact of these systems on various stakeholders (users, providers, community, etc.) needs to be assessed prior to the implementation of these systems.

It is still an open question regarding which travel demand policies have the greatest impact on traffic congestion, while imposing the least social costs. It is the contention of this special issue that new insights will become available because of the interface among different disciplines (e.g. social and behavioral sciences (economics) with engineering (systems and transportation engineering)) that bring together different dimensions of the congestion problem, and integrate different methodologies (e.g. game theory, experimental economics, agent-based theory, mathematical programming, artificial intelligence, dynamic simulation, and modeling among others).

\section{Economic research with linkages to engineering}

Both theoretical and experimental economists have finally developed an interest in urban traffic congestion problems, as witnessed by the lengthy list of papers on the subject. For a long time, traffic congestion was simply considered as belonging to the domain of engineers. Congestion was considered to be a supply side problem, caused by insufficient road capacity. As such, it could be managed by simply building more infrastructure. Demand was taken as given and unchangeable. Surprisingly, the importance of incentives in motivating individuals' actions was not explicitly considered, even after the engineers had formulated the three famous road paradoxes. These paradoxes, indeed, occur exactly because of individuals properly responding to incentives. So we now provide a brief description of these three paradoxes.

The Pigou-Knight-Downs paradox states that expanding road capacity as a remedy to traffic congestion leads to no reductions in travel cost, as new road capacity will elicit new demand. The Down-Thomson paradox additionally claims that expanding road capacity is not only ineffective, but often counterproductive if public transportation is available. As the road service improves and commuters switch from public transportation to the congested road, the metro or bus may run less frequently and the worsened service may induce more commuters to additionally switch, until the benefit of improved road capacity dissipates completely. As a result of the road improvement, in the first paradox, travel costs do not change; while in the second paradox, the system settles into an equilibrium far worse than before, in the sense that in the end travel time increases for all commuters, both on the road and on public transportation. The third paradox is the Braess Paradox, which states that adding a costless link between two alternative routes can actually increase the travel cost for all users. The fact that 'traffic expands to meet the available road space' (Mogridge 1990) was posited as the 'Iron Law of Congestion' by Downs, and extensively documented by Mogridge with the case study of London in his book Travel in Towns: Jam Yesterday, Jam Today and Jam Tomorrow? These paradoxes are the consequence of individuals' equilibrium behavior and individual responses to particular incentives. The actual reaction of policy authorities and the planning tools that should be used to avoid or correct the unanticipated results of the paradoxes are far from clear.

Studying individuals' behavior in network environments is notoriously difficult. As Horowitz (1984) observes, significant differences are often found between actual traffic volumes and traffic volumes as calculated with models. These differences 
could be the result of model misspecification rather than failure of the model itself. Also, because traffic and transportation networks are highly interconnected, it is hard to design a 'natural experiment' to test clearly the equilibrium predictions and confirm the realization of the paradoxes. As a result, a growing number of researchers, particularly economists, have started using controlled laboratory experiments to examine route-choice behavior. In these experiments subjects are called into a laboratory and asked to make choices between different routes. Subjects are then paid according to the rewards associated with the routes chosen. Experimental studies ask whether equilibrium in patterns of traffic flow are realized as individuals interact repeatedly on a network, whether traffic flows adjust in response to network changes and, if so, how changes in traffic condition relates to traffic flows and adjustments.

The commuter's route choice problem has been studied in an interesting experiment by Selten et al. (2007), who find that, despite persistent fluctuations, the mean number of travelers along each route is close to equilibrium. DenantBoemont and Hanmiche (2009) and Mago and Razzolini (2010) study the DownThomson paradox in the laboratory to find that it does indeed hold when new road capacity is introduced. The Mago and Razzolini paper confirms that increased road capacity diverts commuters from the congested road, and this worsens the alternative transit service in a way such that the new equilibrium occurs at a level of congestion higher than before. The paper also finds that the size of the population and the pricing structure of the public transit system matter with respect to coordination on the equilibrium route choice. In recent years Rapoport et al. (2006, 2009) have explored different aspects of this problem in a number of experiments. In particular, they test the Braess paradox in the laboratory, as well as different aspects of how subjects make commuting decisions in an already congested network. Morgan et al. (2009) study both the Braess and the Pigou-Knight-Downs paradox in the laboratory. They consider network changes based on the two paradoxes and find adjustments in traffic flows commensurate with attaining equilibrium (rather than social efficiency). Thus it is clear that while work has begun on these topics there is still a lot that needs to be done - in particular by providing evidence for or against a model, experimental economics lays the first step for developing new theories and better understanding of behavior and congestion issues.

\section{Overview of the five contributions of this special issue}

The five papers selected for this special issue provide novel insights of their own. They cover the modeling of parking behavior using possibility theory as well as the evaluation of a novel concept called Highway Space Inventory Control where drivers must book in advance for their highway usage. Evaluating the implications of new types of traffic pricing schemes continues to be a challenge. One paper in this issue tackles exactly this problem by evaluating different measures using data from the Netherlands. A fourth paper examines the implications of a disaster (bridge collapse) on traffic and assigns an economic value to such an outcome. The final paper is a case study that shows that price-based mechanisms may not be the best way to curb congestion.

The first paper, entitled 'Modelling Parking Choice Behaviour using Possibility Theory,' by M. Ottomanelli, M. Dell'Orco, and D. Sassanelli, provides an example of 
how an engineering approach grounded in possibility theory can offer an evaluation of parking users' behavior. This paper presents a discrete choice model. The approach is an alternative way to represent imperfect knowledge (uncertainty) of users about the status of both the parking and transportation system, in addition to the approximate reasoning of the human decision maker (imprecision). The resulting model is a quantitative soft computing approach that can support traffic analysts in planning future parking policies and the design of an advanced traveler information system (ATIS). The effects of information on user choice can be incorporated in the model itself. Thus, the parking user is considered to be a decision-maker that assumes a certain choice set, that is the set of perceived parking alternatives and has some information about the parking supply system. He/she associates with each parking alternative an approximate perceived cost/utility that is represented by a possibility distribution. The user chooses the alternative that minimizes/maximizes his/her perceived parking cost/utility, respectively. The results of this research show that the proposed approach is able to represent the effect of various parking policies on the behavior of users.

The second paper, entitled 'Evaluating the Effects of Urban Congestion Pricing: Geographical Accessibility versus Social Surplus' by Taede Tillema et al., combines economic principles with geographic considerations. This paper proposes and compares the use of two alternative evaluation measures to assess the effectiveness of road pricing measures, that is a geographical accessibility measure and an economic social surplus measure. The comparison is conducted from a theoretical perspective as well as by studying the associated outcomes of these measures for a research area in the Netherlands. By means of correlation analysis and spatial comparison of outcomes, it is found that geographical accessibility measures are simpler and easier to interpret than the economic measures. However, they offer a poor proxy of the outcomes of the economic evaluation measures. Therefore, the decision as to whether one should use economic surplus measures or geographical accessibility indicators to a large extent depends on the research goal. If the goal is to gain a thorough insight into the monetary gains/losses resulting from a policy measure, economic measures, such as the rule-of-half or the logsum measure, are preferable. However, if there are concrete questions about the changes in accessibility of certain types of activity locations, geographical indicators, such as the contour and potential measures, are more appropriate. The outcomes of such geographical measures, and especially those of the contour measures, are, however, sensitive to the spatial distribution of activity locations in the area under study and to the selection of the impedance parameter that has to be selected in advance in order to compute the effects. Therefore, if geographical indicators are being used, it would be wise to use different sensitivity parameters. It is only then that a thorough insight can be gained into the sensitivity of geographical accessibility effects.

The third paper, entitled 'Evaluating the Effects of I-35W Bridge Collapse on Road-Users in the Twin Cities Metropolitan Region' by F. Xie and D. Levinson, combines engineering principles with economic concepts. This research evaluates the effects of the I-35W bridge collapse on road users in the Minneapolis-St. Paul, Minnesota (Twin Cities) metropolitan area. The Twin Cities Seven-County travel demand model developed in previous research is re-calibrated with July 2007 loop detector traffic data. This re-calibrated model is used to carry out an evaluation of economic loss incurred by increased travel delay in alternative scenarios before and 
after the bridge collapse. It is found that the failure of the bridge resulted in an economic loss of US\$71,000 to US\$220,000 a day, depending on how flexible road users in the system can adjust their trip destinations in response to the bridge closing. It is also estimated that the major traffic restoration projects that the Minnesota DOT has implemented in quick response to the bridge collapse can save road users US\$9,500 to US\$17,500 a day. This translates into a benefit-cost ratio of 2.0-9.0, suggesting these projects are highly beneficial in an economic sense. In this analysis, the use of a simplified, scaled-down travel demand model enables one to carry out the analysis quickly and accurately, which contributes to transportation planning under situations such as emergency relief.

The fourth paper, entitled 'A Simulation-based Methodology to Compare the Performance of Highway Space Inventory Control and Ramp Metering Control' by P. Edara et al., explores the benefits of a novel engineering concept, that is of a highway space inventory control system (HSICS) as a mechanism to mitigate traffic congestion. Within a HSICS all road users have to make reservations in advance to enter the highway. The proposed system allocates highway spaces temporally and spatially over time so as to optimize highway utilization by maximizing the passenger miles of travel. In theory, HSICS offers additional benefits as compared to ramp metering control. Queue lengths on the ramps due to excessive on-ramp demand will be shorter in the case of HSICS because motorists realize that it is not possible to access the highway if they do not have an advanced reservation, and therefore will not wait for their 'free' turn to enter, as they would in the case of ramp metering. Also, drivers with reservations can be assured of more reliable travel times as compared to ramp metering. In this paper, a methodology is developed to perform a quantitative comparison of the operational performance of HSICS and two isolated ramp metering techniques: ALINEA and fixed pre-timed metering. The methodology involves a combination of microscopic traffic simulation and heuristic optimization. The proposed framework is generic and can be applied to compare other traffic control and demand management techniques. The proposed methodology was tested on a simulated highway section with traffic and geometric characteristics similar to a real congested site. Simulation results show that HSICS produces approximately 35 and $45 \%$ more passenger miles than ALINEA and fixed pre-timed metering, respectively. Vehicle miles traveled values for HSICS were comparable, $4 \%$ lower, to those of the ALINEA.

The fifth and last paper of this special issue, entitled 'Auto Restricted Zone versus Price Changes: A Case Study,' by L. Stuart and S. Sarangi, provides an economic evaluation of the Auto Restricted Zone Policy (ARZ). This is a case study that compares ARZ with price changes for parking permits at Louisiana State University (LSU). It is found that ARZ has a more significant effect on the reduction in the purchase of parking permits than price increases, albeit with a lag. It is also found that while driving continues to be the primary mode of choice, a significant number of students are willing to adopt alternative means of transportation if facilities associated with them are improved. Since LSU is fairly representative it is believed that this case study contains lessons for other universities in the country facing traffic congestion issues. 


\section{Acknowledgements}

Support for this special issue was provided by the US National Science Foundation, HSD grants 0527315,0527534 and 0527315 . This paper is based in part on work supported by the National Science Foundation, while working at the Foundation. Any opinions, conclusions, and/or findings are those of the authors and do not necessarily reflect the views of the National Science Foundation.

\section{References}

Denant-Boemont, L. and Hammiche, S., 2009. Public transit capacity and user's choice: an experiment on Downs Thomson Paradox. Proceedings of the 4th Kuhmo-Nectar Conference on Transportation and Urban Economics, Technical University of Denmark, Copenhagen. Available from: http://halshs.archives-ouvertes.fr/docs/00/40/62/2 . .ent_kuhmo-nectar.pdf.

Dupuit, J., 1844. On the measurement of the utility of public works. Translated by R.M. Barback from the "Annales des Ponts et Chaussées," 2nd series, 7 (1844). International Economic Papers, No. 2. London: Macmillan, 1952, 83-110.

Horowitz, J.L., 1984. The stability of stochastic equilibrium in a two-link transportation network. Transportation Research B, 18, 13-28.

Knight, F.H., 1924. Some fallacies in the interpretation of social cost. Quarterly Journal of Economics, 38, 582-606.

Mago, S.D. and Razzolini, L., 2010. An experimental study of traffic congestion and route choice. Mimeo. Richmond, VA: Virginia Commonwealth University.

Mogridge, M.J.H., 1990. Travel in towns: tam yesterday, jam today and jam tomorrow? London: Macmillan Press.

Morgan, J., Orzen, H., and Sefton, M., 2009. Network architecture and traffic flows: experiment on the Pigou-Knight-Down and Braess Paradoxes. Games and Economic Behavior, 66 (1), 348-372.

Pigou, A.C., 1920. The economics of welfare. London: Macmillan.

Putnam, R.D., 2000. Bowling alone: the collapse and revival of American community. New York: Simon \& Schuster.

Rapoport, A., Mak, V., and Zwick, R., 2006. Navigating congested networks with variable demand: experimental evidence. Journal of Economic Psychology, 27, 648-666.

Rapoport, A., et al., 2009. Choice of routes in congested traffic networks: experimental tests of the Braess Paradox. Games and Economic Behavior, 65 (2), 538-571.

Schrank, D. and Lomax, T., 2009. The 2009 urban mobility report. Texas College Station, TX: Transportation Institute, Texas A\&M University.

Selten, R., et al., 2007. Commuters route choice behavior. Games and Economic Behavior, 58 (2), 394-406.

Smeed Report, 1964. Road pricing: the economic and technical possibilities. London: HMSO. Available from: http://www.answers.com/topic/smeed-report [Accessed 1 August 2011].

Teodorovič, D. and Lučić, P., 2005. Intelligent parking systems. European Journal of Operations Research, 175 (3), 1666-1681.

Transport for London, TfL, 2008. Central London congestion charging: impacts monitoring: Sixth Annual Report. London: Transport for London. Available from: http://www.tfl.gov.uk/ assets/downloads/sixth-annual-impacts-monitoring-report-2008-07.pdf [Accessed 1 August 2011].

Transportation Research Part, A, 2009. Special issue on Stockholm congestion charging trial. Transportation Research Part A, 43 (3), 237-309.

Vickrey, W.S., 1969. Congestion theory and transport investment. The American Economic Review, 59 (2), 251-260.

VTPI, 2004. The cost of driving and the savings from reduced vehicle use, 1-12 [online]. Available from: http://www.vtpi.org/tdm/tdm82.htm.

Zhao, Y., et al., 2010. A travel demand management strategy: the downtown space reservation system. European Journal of Operational Research, 205 (3), 584-594. 